\title{
INTEGRAL REPRESENTATIONS FOR CONTINUOUS LINEAR OPERATORS IN THE SETTING OF CONVEX TOPOLOGICAL VECTOR SPACES
}

\author{
BY
}

\author{
J. R. EDWARDS AND S. G. WAYMENT
}

\begin{abstract}
Suppose $X$ and $Y$ are locally convex Hausdorff spaces, $H$ is arbitrary and $\Sigma$ is a ring of subsets of $H$. The authors prove the analog of the theorem stated in [Abstract 672-372, Notices Amer. Math. Soc. 17 (1970), 188] in this setting. A theory of extended integration on function spaces with Lebesgue and non-Lebesgue type convex topologies is then developed. As applications, integral representations for continuous transformations into $Y$ for the following function spaces $F$ (which have domain $H$ and range $X$ ) are obtained: (1) $H$ and $\Sigma$ are arbitrary, $\tau$ is a convex topology on the simple functions over $\Sigma, K$ is a set function on $\Sigma$ with values in $L[X, Y]$, and $F$ is the Lebesgue-type space generated by $K$; (2) $H$ is a normal space and $F$ is the space of continuous functions each of whose range is totally bounded, with the topology of uniform convergence; (3) $H$ is a locally compact Hausdorff space, $F$ is the space of continuous functions of compact support with the topology of uniform convergence; (4) $H$ is a locally compact Hausdorff space and $F$ is the space of continuous functions with the topology of uniform convergence on compact subsets. In the above $X$ and $Y$ may be replaced by topological Hausdorff spaces under certain additional compensating requirements.
\end{abstract}

1. Introduction. Let $H$ be a compact Hausdorff space, and let $X$ and $Y$ be locally convex topological vector spaces over the real or complex field where $Y$ is Hausdorff. Let $C(H, X)$ be the space of continuous functions from $H$ into $X$ with the topology of uniform convergence. R. K. Goodrich [6] has given an integral representation theorem for a continuous linear transformation $T$ from $C(H, X)$ into $Y$. In this paper we extend the aforementioned theorem to the setting where $H$ is an arbitrary space and $C(H, X)$ is replaced by a function space $F$ which is a subset of the closure of the totally bounded functions from $H$ into $X$ under a locally convex topology $\tau$ not stronger than the topology of uniform convergence and satisfying certain extension properties relative to simple functions. The operator valued measures which arise in connection with these integral representations are used to generate Lebesgue-type topologies on $F$ and then a representation theorem is given for transformations $T$ from $F$ into a topological space $Z$ under these new

Presented in part to the Society, January 24, 1970 under the title A unifying representation theorem; received by the editors January 19, 1970 and, in revised form, September 15, 1970.

AMS 1969 subject classifications. Primary 2850, 4725; Secondary 4601.

Key words and phrases. Integral representations, linear operators, locally convex topologies, Lebesgue-type topologies, extended integrals, vector valued functions, vector valued set functions, quasi-Gowurin, bounded $(p, q)$ variation, weak convergence.

Copyright (C) 1971, American Mathematical Society 
topologies. These results generalize the works of Easton and Tucker [2] who give a Lebesgue-type integral and a representation theorem for the associated function space in the setting where $H$ is compact, $X$ and $Y$ are normed spaces, and $K$ is of bounded semivariation.

As a corollary we restrict considerations to the setting where $H$ is normal and $F$ is the space of continuous totally bounded functions with the topology of uniform convergence, which extends the results in [3]. Another corollary gives a representation for continuous operators on the space of continuous functions under the topology of uniform convergence on compact subsets where the domain space $H$ of the functions is locally compact.

This paper continues the development in [3] where $\dot{X}$ and $Y$ were assumed to be normed spaces.

2. Notation and preliminaries. A linear map $T$ from $X$ into $Y$ is $(p, q)$ related if for each continuous seminorm $q$ on $Y$ there is a continuous seminorm $p$ on $X$ and a constant $L_{p, q}$ such that $q(T(x)) \leqq L_{p, q} p(x)$. Furthermore if $T$ is $(p, q)$ related we define $|T|_{p, q}=\sup _{x \in X}\{q(T(x)): p(x) \leqq 1\}$. By $X^{+}$we mean the weak sequential completion of $X$, i.e., the linear subspace of $X^{\prime \prime}$ (the bidual of $X$ ) such that $x^{+} \in X^{+}$ if and only if there is a sequence in $X$ which converges weakly to $x^{+}$. The topology on $X^{+}$is the topology of uniform convergence on equicontinuous subsets of $X^{\prime}$ (the continuous dual of $X$ ), i.e., the $\varepsilon^{00}$ topology. We shall let $X^{\omega}$ denote the subspace of $X^{\prime \prime}$ which is the weak closure of $X$. Again the topology is the $\varepsilon^{00}$ topology.

The development of most of this paper could be carried out with a field of sets $\Sigma \subset P(H)$ and integration in the Stieltjes-Hildebrandt sense [7], i.e., a function $f$ from $H$ into $X$ is said to be integrable with respect to the finitely additive set function $K$ from $\Sigma$ into $L[X, Y]$ (the space of bounded linear operators from $X$ into $Y$ ) if $\lim _{\pi} \sum\left[K\left(E_{i}^{\pi}\right)\right]\left(f\left(t_{i}^{\pi}\right)\right)$ exists in $\bar{Y}$, the completion of $Y$, where $\left\{E_{i}^{\pi}\right\}$ is a partition of $H$ over $\Sigma$ and $t_{i}^{\pi} \in E_{i}^{\pi}$, and where the direction on the net of partitions is refinement. However, Theorems 6.2 and 6.3 require a slight extension of the notion of integration which we now make. Let $\Sigma \subset P(H)$ denote a ring of sets rather than a field. We shall say $\pi=\left\{E_{i}^{\pi}\right\}_{i=1}^{n}$ is a partition of $H$ over $\Sigma$ provided $\left\{E_{i}^{\pi}\right\}_{i=1}^{n-1}$ is a collection of pairwise disjoint sets in $\Sigma$ and $E_{n}=\sim \bigcup_{i=1}^{n-1} E_{i}$. The collection of partitions of $H$ over $\Sigma$ is directed by $\pi=\left\{E_{i}\right\}_{i=1}^{n}>\pi^{\prime}=\left\{F_{j}\right\}_{j=1}^{m}$ if and only if $\bigcup_{j=1}^{m-1} F_{j} \subset \bigcup_{i=1}^{n-1} E_{i}$ and $E_{i} \cap F_{j} \neq 0$ implies $E_{i} \supset F_{j}$. A function $f$ is integrable provided $\lim _{\pi} \sum_{i=1}^{n-1}\left[K\left(E_{i}^{\pi}\right)\right]\left(f\left(t_{i}^{\pi}\right)\right)$ exists in $\bar{Y}$.

Let $S(\Sigma, X)$ denote the linear space of $X$-valued simple functions on $H$ over $\Sigma$, i.e., functions of the form $\sum_{i=1}^{n} \chi_{E_{i}} \cdot x_{i}$ where $E_{i} \in \Sigma, i=1, \ldots, n-1, E_{n}=\sim \bigcup_{i=1}^{n-1} E_{i}$, the complement of $\bigcup_{i=1}^{n-1} E_{i}$, and where $x_{n}=\theta_{X}$. Suppose $\tau$ is a topology on $S(\Sigma, X)$ under which it is a topological vector space. Then a finitely additive set function $K$ from $\Sigma$ into $L[X, Y]$ is said to be quasi-Gowurin with respect to $\tau$ if given a neighborhood $V$ of $\theta_{Y}$ there is a neighborhood $U$ of $\theta_{S(\Sigma, X)}$ such that if $\sum \chi_{E_{i}} \cdot x_{i} \in U$, then $\sum\left[K\left(E_{i}\right)\right]\left(x_{i}\right) \in V$. If $\tau$ is locally convex and $K$ is quasi-Gowurin, then for each continuous seminorm $q$ on $Y$ there is a continuous seminorm $\rho$ on $S(\Sigma, X)$ 
and a smallest constant $W K_{\rho, q}$ such that $q\left(\sum\left[K\left(E_{i}\right)\right]\left(x_{i}\right)\right) \leqq W K_{\rho, q} \rho\left(\sum \chi_{E_{i}} \cdot x_{i}\right)$. If $X$ is a locally convex topological vector space and $\tau$ is the topology of uniform convergence, then quasi-Gowurin becomes bounded $\left(p, q^{\prime \prime}\right)$ variation [6]. We shall use all other definitions and notations defined in [6], except that we shall view $X \subset X^{+} \subset X^{\omega} \subset X^{\prime \prime}$, where $X^{\omega}$ denotes the weak closure of $X$.

Let $\Gamma$ denote the closure of $S(\Sigma, X)$ under the topology of uniform convergence. The following lemma is straightforward and is stated without proof.

2.1 Lemma. If $f \in \Gamma$, then $\lim _{\pi} \sum \chi_{E_{i}^{\pi}} \cdot f\left(t_{i}^{\pi}\right)=f$, where the convergence is uniform.

2.2 THEOREM. Suppose the linear subspace $F$ is contained in $\Gamma$ and $\tau$ is a locally convex topology on $F+S(\Sigma, X)$ which is not stronger than the topology of uniform convergence. If $K$ is quasi-Gowurin with respect to $\tau$, then the linear operator $T(f)$ $=\int d K \cdot f$ exists for each $f \in F$ and is a continuous operator on $F+S(\Sigma, X)$. Furthermore, if $T$ is $(\rho, q)$ related then $W K_{\rho, q}=|T|_{\rho, q}$.

Proof. Suppose $f \in F$ and $q$ is a continuous seminorm on $\bar{Y}$. Then there is a continuous seminorm $\rho$ on $F+S(\Sigma, X)$ such that $K$ is $(\rho, q)$ related. Then for partitions $\pi$ and $\pi^{\prime}$,

$$
q\left(\sum_{\pi}\left[K\left(E_{i}\right)\right]\left(f\left(t_{i}\right)\right)-\sum_{\pi^{\prime}}\left[K\left(E_{j}\right)\right]\left(f\left(t_{j}\right)\right)\right) \leqq W K_{\rho, q} \rho\left(\sum_{\pi} \chi_{E_{i}} \cdot f\left(t_{i}\right)-\sum_{\pi} \chi_{E} \cdot f\left(t_{j}\right)\right) .
$$

Since $\Gamma$ is not stronger than the topology of uniform convergence, it follows that $\rho$ is continuous on $S(\Sigma, X)$ with the topology of uniform convergence. It follows from Lemma 2.1 that $\left\{\sum_{\pi} \chi_{E_{i}} \cdot f\left(t_{i}\right)\right\}_{\pi}$ is Cauchy in the topology of uniform convergence. Therefore, $\left\{q\left(\sum_{\pi}\left[K\left(E_{i}\right)\right]\left(f\left(t_{i}\right)\right)\right\}_{\pi}\right.$ is Cauchy and hence $\left\{\sum_{\pi}\left[K\left(E_{i}\right)\right]\left(f\left(t_{i}\right)\right)\right\}_{\pi}$ is Cauchy in $Y$, from which it follows that $\int d K \cdot f$ exists in $\bar{Y}$. Furthermore,

$$
\begin{aligned}
q\left(\int d K \cdot f\right) & =\lim _{\pi} q\left(\sum_{\pi}\left[K\left(E_{i}\right)\right]\left(f\left(t_{i}\right)\right)\right) \\
& \leqq \lim _{\pi} \sup W K_{\rho, q} \rho\left(\sum_{\pi} \chi_{E_{i}} \cdot f\left(t_{i}\right)\right) \\
& =W K_{\rho, q} \rho(f) .
\end{aligned}
$$

Hence $T$ is $(\rho, q)$ related and $|T|_{\rho, q} \leqq W K_{\rho, q}$, from which we conclude $T$ is continuous. Finally, we show $|T|_{\rho, q} \geqq W K_{\rho, q}$. Suppose $\left\{E_{i}\right\}=\pi$ is a partition of $H$ over $\Sigma$, and $\left\{x_{i}\right\} \subset X$. Then

$$
q\left(\sum\left[K\left(E_{i}\right)\right]\left(x_{i}\right)\right)=q\left(T\left(\sum \chi_{E_{i}} \cdot x_{i}\right)\right) \leqq|T|_{\rho, q} \rho\left(\sum \chi_{E_{i}} \cdot x_{i}\right) .
$$

2.3 Corollary. Suppose $F, S(\Sigma, X), \tau$ and $K$ are as in Theorem 2.2. If $\left\{f_{\alpha}\right\}$ $\subset(F+S(\Sigma, X))$ is a net converging to $f \in F+S(\Sigma, X)$, then $\int d K \cdot f_{\alpha} \rightarrow \int d K \cdot f$.

3. The main result. Suppose $F \subset \Gamma$ is a locally convex topological space with topology $\tau$ not stronger than the topology of uniform convergence and such that there is a ring of sets $\Sigma$ and a subspace $S^{\prime}(\Sigma, X) \subset S(\Sigma, X)$ satisfying: 
(i) The topology $\tau$ has a locally convex extension to $F+S(\Sigma, X)$.

(ii) $F$ is contained in the $\tau$ closure of $S^{\prime}(\Sigma, X)$.

(iii) There is a linear map $\eta$ from $F+S(\Sigma, X)$ into $F^{\omega}$ which is $\left(\rho, \rho^{\prime \prime}\right)$ related when restricted to $S(\Sigma, X)$, and continuous when restricted to $F+S^{\prime}(\Sigma, X)$ and such that $\eta(f)=f$ for each $f \in F$.

Since $\eta$ when restricted to $S(\Sigma, X)$ is $\left(\rho, \rho^{\prime \prime}\right)$ related, for each continuous seminorm $\rho$ there is a constant $|\eta|_{\rho}$ such that $\rho^{\prime \prime}\left(\eta\left(\sum_{\pi} \chi_{E_{i}} \cdot x_{i}\right)\right) \leqq|\eta|_{\rho} \rho\left(\sum_{\pi} \chi_{E_{i}} \cdot x_{i}\right)$.

3.1 Theorem. Suppose $T$ is a continuous linear transformation from $F$ into $Y$. Then, there is an additive set function $K$ on $\Sigma$ with values in $L\left[X, Y^{\omega}\right]$ which is quasiGowurin with respect to $\tau$ such that $T(f)=\int d K \cdot f$. Furthermore, if $T$ is $(\rho, q)$ related, then $W K_{\rho, q^{\prime \prime}} \geqq|T|_{\rho, q} \geqq W K_{\rho, q^{\prime \prime}} /|\eta|_{\rho}$.

Proof. Let $T^{\omega}$ denote the continuous linear map from $F^{\omega}$ into $Y^{\omega}$ which is the natural extension of $T$. Define the set function $K$ from $\Sigma$ into $L\left[X, Y^{\omega}\right]$ by $[K(E)](x)=T^{\omega}\left(\eta\left(\chi_{E} \cdot x\right)\right)$ for $x \in X$ and for each $E \in \Sigma$. Suppose $q$ is a continuous seminorm on $Y$. Since $T^{\omega} \eta$ is continuous on $S(\Sigma, X)$, there is a continuous seminorm $\rho$ on $F+S(\Sigma, X)$ such that, for $\pi=\left\{E_{i}\right\}$ a partition of $H$ and a corresponding collection $\left\{x_{i}\right\} \subset X$,

$$
\begin{aligned}
q^{\prime \prime}\left(\sum_{\pi}\left[K\left(E_{i}\right)\right]\left(x_{i}\right)\right) & =q^{\prime \prime}\left(T^{\omega}\left(\eta\left(\sum \chi_{E_{i}} \cdot x_{i}\right)\right)\right) \\
& \leqq\left|T^{\omega}\right|_{\rho^{\prime \prime}, q^{\prime \prime}}|\eta|_{\rho}\left(\sum \chi_{E_{i}} \cdot x_{i}\right) .
\end{aligned}
$$

From which it follows that $K$ is quasi-Gowurin with respect to $\tau$ and that $W K_{\rho, q^{\prime \prime}}|\eta|_{\rho} \leqq|T|_{\rho, q}$, since $\left|T^{\omega}\right|_{\rho^{\prime \prime}, q^{\prime \prime}}=|T|_{\rho, q}$. Suppose $f \in F$. Then (ii) implies there is a net $\left\{s_{\alpha}\right\}$ in $S^{\prime}(\Sigma, X)$ which converges to $f$ in the $\tau$ topology. Since $\eta$ is continuous on $F+S^{\prime}(\Sigma, X)$, it follows from Corollary 2.3 that

$$
T(f)=T^{\omega}(\eta(f))=\lim _{\alpha} T^{\omega}\left(\eta\left(s_{\alpha}\right)\right)=\lim _{\alpha} \int d K \cdot s_{\alpha}=\int d K \cdot f .
$$

Theorem 2.2 implies that if $T$ is $(\rho, q)$ related, then $|T|_{\rho, q} \leqq W K_{\rho, q^{\prime \prime}}$, and the theorem is established.

The following lemma gives a necessary and sufficient condition for $\eta$ to be continuous on $F+S^{\prime}(\Sigma, X)$. The proof is analogous to that given for Corollary 2.3 in [3] and is omitted.

3.2 Lemma. Suppose the linear map $\eta$ from $F+S^{\prime}(\Sigma, X)$ into $F^{\omega}$ satisfies the condition that $\eta(f)=f$ for each $f \in F$. Then a necessary and sufficient condition that $\eta$ be continuous on $F+S^{\prime}(\Sigma, X)$ is that if the net $\left\{s_{\alpha}\right\} \subset S^{\prime}(\Sigma, X)$ converges to $f \in F$ in the $\tau$ topology then $\left\{\eta\left(s_{\alpha}\right)\right\}$ converges to fin the $\tau^{\omega}$ topology (the $\varepsilon^{00}$ topology restricted to $\left.F^{\omega}\right)$.

3.3 REMARK. If the map $\eta$ takes its values in $F^{+}$, then the $K$ given in Theorem 3.1 takes its values in $L\left[X, Y^{+}\right]$. Similarly, if $\eta$ takes its values in $F$, then $K$ takes its values in $L[X, Y]$. 
4. The extended integral. In this section we develop a Lebesgue-type extension of the integral and give an extended version of Theorem 3.1. We suppose the general setting of $\S 3$. Let $G$ denote the $\tau$ completion of $F$. Observe that $G$ is a collection of equivalence classes of nets. The question of when it is possible to identify functions from $H$ into $X$ with equivalence classes is of some interest. In the following three definitions we define certain classes of functions which have natural identifications with equivalence classes in $G$.

4.1 Definition. Let $G(\tau)$ denote the space of functions $f$ on $H$ with values in $X$ such that all nets of simple functions of the form $\left\{\sum_{\pi} \chi_{E_{i}} \cdot f\left(t_{i}\right)\right\}_{\pi}$, where $t_{i} \in E_{i}$, are Cauchy and Cauchy equivalent in the $\tau$ topology.

Observe that $\Gamma \subset G(\tau)$ because the topology of uniform convergence is stronger than $\tau$. The identification of $G(\tau)$ as a subspace of $G$ is the natural one.

4.2 Definition. A function $f$ on $H$ with values in $X$ is said to be an allowable $\sigma$-totally bounded function if its range is the union of a nested countable collection of sets $\left\{B_{n}\right\}_{n=1}^{\infty}$ ordered as to $n$, such that each $B_{n}$ is totally bounded and such that $\chi_{f^{-1}}{ }_{\left[B_{n}\right]} \cdot f \in \Gamma$. The collection $\left\{H_{n}\right\}=\left\{f^{-1}\left[B_{n}\right]\right\}$ is called a $\sigma$-totally bounded decomposition of $H$ with respect to $f$ (we assume $H_{n} \in \Sigma$ for each $n$ ).

Let $\Lambda$ denote the directed set $\left\{(V, n): V\right.$ is a neighborhood of $\theta_{\Gamma}$ and $n$ is an integer $\}$ whose direction is given by $(V, n)>(U, m)$ if $U \supset V$ and $n \geqq m$.

4.3 Definition. Let $G^{\sigma}(\tau)$ denote the space of allowable $\sigma$-totally bounded functions such that if $\left\{H_{n}\right\}$ and $\left\{F_{n}\right\}$ are two $\sigma$-totally bounded decompositions of $H$ with respect to $f$ and if $\left\{s_{\alpha}\right\}_{\Lambda}$ and $\left\{t_{\alpha}\right\}_{\Lambda}$ are nets of functions in $F$ such that for $\alpha=(V, n), s_{\alpha}-\chi_{H_{n}} \cdot f$ and $t_{\alpha}-\chi_{F_{n}} \cdot f$ are in $V$, then $\left\{s_{\alpha}\right\}$ and $\left\{t_{\alpha}\right\}$ are Cauchy and Cauchy equivalent.

For each $f \in G^{\sigma}(\tau)$ we identify $f$ with the equivalence class in $G$ determined by Definition 4.3.

4.4 Definition. Suppose $K$ is quasi-Gowurin with respect to $\tau$. Then, for $f \in G$ the extended integral of $f$ with respect to $K$ is denoted by $E \int d K \cdot f$ and is defined by $\lim _{\alpha} \int d K \cdot f_{\alpha} \in \bar{Y}$ where $\left\{f_{\alpha}\right\} \subset F$ is a net which converges to $f$ in the $\tau$ topology.

The following lemma guarantees that the extended integral is well defined and is consistent with the integral on $G(\tau)$.

4.5 Lemma. Suppose that $K$ is quasi-Gowurin with respect to $\tau$. Then,

(i) if $\left\{f_{\alpha}\right\}$ and $\left\{g_{\alpha}\right\}$ are Cauchy equivalent in $F$, then $\left\{\int d K \cdot f_{\alpha}\right\}$ and $\left\{\int d K \cdot g_{\alpha}\right\}$ are Cauchy equivalent,

(ii) if $f \in G$, the extended integral $E \int d K \cdot f$ exists,

(iii) if $f \in G(\tau)$, then $E \int d K \cdot f=\int d K \cdot f$.

The proof of this lemma follows from Theorem 2.2.

4.6 THEOREM. Suppose $K$ is a finitely additive set function on $\Sigma$ with values in $L[X, Y]$ which is quasi-Gowurin with respect to $\tau$. Then the map $T(f)=E \int d K \cdot f$ is a continuous linear operator from $G$ into $\bar{Y}$. Furthermore, if $T$ is $(\rho, q)$ related, then $|T|_{\rho, q}=W K_{\rho, q}$ 
The proof follows from Theorem 2.2 and Lemma 4.5.

4.7 Theorem. Suppose $T$ is a continuous linear operator from $G$ into $Y$. Then, there is a finitely additive set function $K$ on $\Sigma$ with values in $L\left[X, Y^{\omega}\right]$ which is quasiGowurin with respect to $\tau$ such that $T(f)=E \int d K \cdot f$ for $f \in G$. Furthermore, if $T$ is $(\rho, q)$ related, then $W K_{\rho, q^{\prime \prime}} \geqq|T|_{\rho, q} \geqq\left. W K_{\rho, q^{\prime \prime}}|| \theta\right|_{\rho}$.

Proof. Since $T$ is a continuous linear operator from $F$ into $Y$, it follows from Theorem 3.1 that there is a set function $K$ which satisfies the above conditions such that $T(f)=\int d K \cdot f$ for $f \in F$. Since $T$ is continuous, it follows from Lemma 4.5(ii) that $T(f)=E \int d K \cdot f$ for all $f \in G$.

4.8 REMARK. If the $\eta$ map of $\S 3$ takes its values in $F^{+}$or $F$ then $K$ in Theorem 4.7 takes its values in $L\left[X, Y^{+}\right]$or $L[X, Y]$ respectively.

5. A Lebesgue-type topology. In this section we define a Lebesgue-type topology on a space of simple functions and complete this space into what we shall call $L_{K}$, the analog of the Lebesgue integrable functions in the classical setting. A characterization of the continuous linear operators from $L_{K}$ into a complete locally convex space is then given. In the special case that the topology $\tau$ considered in this section is the topology of uniform convergence, we have an extension of the results in [2] and of the corresponding results in [3] which are given in the setting of linear normed spaces.

Let $X, Y$, and $H$ be as in $\S 1$. Let $\Sigma \subset P(H)$ be a ring of sets and let $\tau$ denote a locally convex topology on $S(\Sigma, X)$ which is not stronger than the topology of uniform convergence. Suppose $K$ is a finitely additive set function on $\Sigma$ with values in $L[X, Y]$ which is quasi-Gowurin with respect to $\tau$. For each continuous seminorm $q$ on $Y$ define the seminorm $\rho_{q}$ on $S(\Sigma, X)$ by

$$
\rho_{q}\left(\sum_{i} \chi_{E_{i}} \cdot x_{i}\right)=\sup \left\{q\left(\sum_{i j}\left[K\left(E_{i} \cap F_{j}\right)\right]\left(\alpha_{j} x_{i}\right)\right)\right\}
$$

where the supremum is taken over all partitions $\left\{F_{j}\right\}$ of $H$ over $\Sigma$ and corresponding collections of scalars $\left\{\alpha_{j}\right\}$ such that $\left|\alpha_{j}\right| \leqq 1$ for each $j$. Since $\tau$ is not stronger than the topology of uniform convergence the supremum exists for each $q$. Two simple functions $s$ and $s^{\prime}$ are said to be equivalent if $\rho_{q}\left(s-s^{\prime}\right)=0$ for each continuous seminorm on $Y$. Let $S(\Sigma, X)_{L}$ denote the resulting space.

5.1 Definition. Let $\kappa$ denote the locally convex topology generated on $S(\Sigma, X)_{L}$ by the seminorms $\rho_{q}$. Let $L_{K}$ denote the completion of $S(\Sigma, X)_{L}$ under the $\kappa$ topology. As is the usual practice, if a function is identified with an equivalence class in $L_{K}$, we do not distinguish between the function and the equivalence class. In this setting we give a more general criterion than those given in $\$ 4$ with which to identify a function with an equivalence class in $L_{K}$. Suppose $f$ is an allowable $\sigma$-totally bounded function on $H$ with values in $X$. If there is a $y \in \bar{Y}$ such that for each $\sigma$-totally bounded decomposition $\left\{H_{n}\right\}$ of $H, \lim _{n} \int_{H_{n}} d K \cdot f=y$, then $f$ is viewed as being in $L_{K}$ and is identified with the equivalence class 
containing the net of simple functions $\left\{s_{\alpha}\right\}_{\Lambda}$ (where $\Lambda$ is defined as in $\S 4$ ) defined by $s_{\alpha}=\sum \chi_{E_{i}^{\alpha}} \cdot f\left(t_{i}\right)$ for $\alpha=(V, n)$ and where $\left\{E_{i}\right\}$ is a partition of $H_{n}$ over $\Sigma$ such that $f\left[E_{i}^{\alpha}\right]-f\left(t_{i}\right) \subset V$, with $t_{i} \in E_{i}$.

5.2 LeMMA. The topology of uniform convergence on $S(\Sigma, X)$ is stronger than the $\kappa$ topology on $S(\Sigma, X)$.

Proof. Suppose $q$ is a continuous seminorm on $Y$. Since $K$ is quasi-Gowurin with respect to $\tau$, there is a seminorm $\rho$ continuous in the $\tau$ topology such that, for $s=\sum \chi_{E_{i}} \cdot x_{i} \in S(\Sigma, X)$,

$$
\begin{aligned}
\rho_{q}(s) & =\sup \left\{q\left(\sum_{i j}\left[K\left(E_{i} \cap F_{j}\right)\right]\left(\alpha_{j} x_{i}\right)\right)\right\} \\
& \leqq \sup \left\{W K_{\rho, q} \rho\left(\sum_{i j} \chi_{E_{i} \cap F_{j}} \alpha_{j} x_{i}\right)\right\}
\end{aligned}
$$

Since $\tau$ is not stronger than the topology of uniform convergence there is a continuous seminorm $p$ on $X$ such that the corresponding seminorm $\rho_{p}$ on $S(\Sigma, X)$ dominates $\rho$. Therefore,

$$
\begin{aligned}
\rho_{q}(s) & \leqq \sup \left\{W K_{\rho, q} \rho_{p}\left(\sum_{i j} \chi_{E_{i} \cap F_{j}} \alpha_{j} x_{i}\right)\right\} \\
& \leqq W K_{\rho, q} \max _{i} p\left(x_{i}\right)=W K_{\rho, q} \rho_{p}(s) .
\end{aligned}
$$

Hence for $f \in \Gamma$ and $q$ a continuous seminorm on $Y, \rho_{q}(f)=\sup \left\{q\left(\sum \alpha_{i} \int_{E_{i}} d K \cdot f\right)\right\}$ where the supremum is taken over partitions $\left\{E_{i}\right\}$ of $H$ over $\Sigma$ and corresponding scalars $\left\{\alpha_{i}\right\}$ such that $\left|\alpha_{i}\right| \leqq 1$ for all $i$. By $\int_{E} d K \cdot f$ we mean $\int d K\left(f \cdot \chi_{E}\right)$ which is well defined for $f \in \Gamma$. Hence, it follows that if $f \in L_{K}$, and if $q$ is a continuous seminorm on $Y$, then $\rho_{q}(f)=\sup \left\{q \sum \alpha_{i} E \int_{E_{i}} d K \cdot f\right\}$.

Suppose $Z$ is a locally convex space. In the following definition we give the analog of the concept of strongly Lipschitz in [2].

5.3 Definition. A set function $G$ on $\Sigma$ with values in $L[X, Z]$ is said to be $(q, v)$ strongly Lipschitz with respect to $K$ if given a partition $\left\{E_{i}\right\}$ of $H$ over $\Sigma$ and a corresponding collection $\left\{x_{i}\right\} \subset X$, then there is a pairing $(q, v)$ with constants $P_{q, v}$ such that

$$
\nu\left(\sum_{i}\left[G\left(E_{i}\right)\right]\left(x_{i}\right)\right) \leqq P_{q, v} \sup \left\{\sum_{i j}\left[K\left(E_{i} \cap F_{j}\right)\right]\left(\alpha_{j} x_{i}\right)\right\}
$$

where the supremum is taken over partitions $\left\{F_{j}\right\}$ of $H$ over $\Sigma$ and corresponding collections of scalars $\left\{\alpha_{j}\right\}$ for which $\left|\alpha_{j}\right| \leqq 1$.

5.4 LeMma. A set function $G$ on $\Sigma$ with values in $L[X, Z]$ is $(q, v)$ strongly Lipschitz if and only if $G$ is quasi-Gowurin with respect to $\kappa$. Furthermore, if $G$ is $(q, v)$ related, then $W G_{\rho_{q}, v}=P_{q, v}$.

The proof is straightforward and is therefore omitted. 
5.5 Theorem. Suppose that $T$ is a continuous linear operator from $L_{K}$ into $Z$. Then, there is an additive set function $G$ on $\Sigma$ with values in $L[X, Z]$ which is $(q, v)$ strongly Lipschitz with respect to $K$ such that for $f \in L_{K}, T(f)=E \int d G \cdot f$. Furthermore, if $T$ is $\left(\rho_{q}, \nu\right)$ related, then $|T|_{\rho_{q}, v}=P_{q, v}$.

Proof. The theorem follows from Theorem 4.7, Remark 4.8, and Lemma 5.4.

If $Z$ is a complete locally convex space, then the above theorem and Theorem 4.6 yield the following characterization of the bounded linear operators from $L_{K}$ into $Z$.

5.6 Corollary. Suppose $T$ is a linear transformation from $L_{K}$ into $Z$. Then $T$ is continuous if and only if there is a finitely additive set function $G$ which is $(q, v)$ strongly Lipschitz with respect to $K$ such that $T(f)=E \int d G \cdot f$ for $f \in L_{K}$. Furthermore, if $T$ is $\left(\rho_{q}, \nu\right)$ related, then $|T|_{\rho_{q}, v}=P_{q, v}$.

6. Some other applications. As before $X$ and $Y$ shall denote locally convex spaces. In $\S \S 7,8$ and 9 we shall establish the following special cases of Theorems 3.1 and 4.7 which in the classical settings have been of particular interest. For $H$ an arbitrary normal space we shall let $C T B$ denote the space of totally bounded continuous functions from $H$ into $X$ with the topology of uniform convergence and shall let $C B R$ denote the space of bounded continuous real valued functions with the topology of uniform convergence. If $H$ is a locally compact Hausdorff space $C_{c}$ denotes the space of continuous $X$-valued functions of compact support and $C_{0}$ denotes the space of continuous $X$-valued functions which vanish at infinity, both with the topology of uniform convergence. The space of continuous realvalued functions of compact support with the topology of uniform convergences is denoted by $C_{c} R$. Finally, if $H$ is locally compact, $C$ shall denote the space of continuous $X$-valued functions from $H$ with the topology of uniform convergence on compact subsets of $H$.

6.1 TheOREM. Let $H$ denote a normal topological space and let $\Sigma$ denote the field of sets generated by the closed subsets of $H$. If $T$ is a continuous linear operator from $C T B$ into $Y$, then there is a unique finitely additive weakly regular set function $K$ on $\Sigma$ with values in $L\left[X, Y^{\omega}\right]$ which is of bounded $\left(p, q^{\prime \prime}\right)$ variation such that $T(f)$ $=\int d K \cdot f$. Furthermore, if $T$ is $(p, q)$ related, then $W K_{p, q^{\prime \prime}}=|T|_{p, q}$.

When we say $T$ is $(p, q)$ related we mean $T$ is $\left(\rho_{p}, q\right)$ related where $\rho_{p}$ is the continuous seminorm on $C T B$ generated by the continuous seminorm $p$ on $X$.

For the purpose of the next theorem we extend the notion of bounded $(p, q)$ variation to additive set functions defined over a ring as follows: The finitely additive set function $K$ from the ring $\Sigma$ into $L[X, Y]$ is of bounded $(p, q)$ variation if there is a pairing $(p, q)$ such that, for each partition $\left\{E_{i}\right\}_{i=1}^{n}$ of $H$ over $\Sigma$ and each collection $\left\{x_{i}\right\}_{i=1}^{n}$ with $x_{n}=\theta, q\left(\sum_{i=1}^{n}\left[K\left(E_{i}\right)\right]\left(x_{i}\right)\right) \leqq L \max _{1 \leqq i \leqq n} p\left(x_{i}\right)$ where the constant $L$ depends on $p$ and $q$. Furthermore, the $(p, q)$ variation of $K$, denoted by $W K_{p, q}$, is given by the infimum of all such $L$. 
6.2 ThEOREM. Let $H$ denote a locally compact Hausdorff space and let $\Sigma_{c}$ denote the ring generated by all compact subsets of $H$. If $T$ is a conitinuous linear operator from $C_{c}$ into $Y$, then there is a unique finitely additive weakly regular set function $K$ on $\Sigma_{c}$ with values in $L\left[X, Y^{\omega}\right]$ which is of bounded $\left(p, q^{\prime \prime}\right)$ variation such that $T(f)$ $=\int d K \cdot f$ for all $f \in C_{c}$. Furthermore, if $T$ is $(p, q)$ related then $W K_{p, q^{\prime \prime}}=|T|_{p, q}$.

6.3 TheOREM. Let $H$ and $\Sigma_{c}$ be as in Theorem 6.2. If $T$ is a continuous linear operator from $C_{0}$ into $Y$, then there is a unique finitely additive weakly regular set function $K$ on $\Sigma_{c}$ with values in $L[X, Y]$ which is of bounded $\left(p, q^{\prime \prime}\right)$ variation such that $T(f)=E \int d K \cdot f$ for $f \in C_{0}$. Furthermore, if $T$ is $(p, q)$ related, then $W K_{p, q^{\prime \prime}}=$ $|T|_{p, q}$.

6.4 Definition. Suppose $\Sigma_{c}$ is as above and $K$ is a finitely additive set function on $\Sigma_{c}$ with values in $L[X, Y]$. To say $K$ is of bounded $(p, q)$ variation with compact $q$-support means, given a continuous seminorm $q$ on $Y$, there are a continuous seminorm $p$ on $X$, a constant $W K_{p, q}$, and a compact set $M_{q}$ such that, for each $x$, $q([K(E)](x))$ is a real-valued set function with support $M_{q}$ and such that $q\left(\sum\left[K\left(E_{i}\right)\right]\left(x_{i}\right)\right) \leqq W K_{p, q} \max \left\{p\left(x_{i}\right): E_{i} \cap M_{q} \neq \varnothing\right\}$. Such a set function is said to be of bounded $(p, q)$ variation with compact support if the compact set $M_{q}$ is independent of the seminorm $q$.

6.5 THEOREM. Let $H$ and $\Sigma_{c}$ be as in Theorem 6.2. If $T$ is a continuous linear operator from $C$ into $Y$, then there is a unique finitely additive weakly regular set function $K$ on $\Sigma_{c}$ with values in $L\left[X, Y^{\omega}\right]$ which is of bounded $\left(p, q^{\prime \prime}\right)$ variation with compact $q^{\prime \prime}$-support such that for $f \in C, T(f)=E \int d K \cdot f$. Furthermore, if $T$ is $(p, q)$ related, then $W K_{p, q^{\prime \prime}}=|T|_{p, q}$.

6.6 TheOREM. Suppose $H$ and $\Sigma_{c}$ are as in Theorem 6.2 and $Y$ is a normed space. If $T$ is a continuous linear operator from $C$ into $Y$, then there is a unique finitely additive weakly regular set function $K$ on $\Sigma_{c}$ with values in $L\left[X, Y^{\omega}\right]$ which is of bounded $\left(p,\|\cdot\|_{Y^{\omega}}\right)$ variation with compact support such that for $f \in C, T(f)=\int d K \cdot f$. Furthermore, if $T$ is $\left(p,\|\cdot\|_{Y^{\omega}}\right)$ related then $W K_{p,\|\cdot\|_{Y} \omega}=|T|_{p,\|\cdot\|_{Y}{ }^{\omega}}$.

We conclude this section by observing that the results obtained in $\$ 3$ of [3] which include generalizations of the results of Hildebrandt [7] and Fihtengol'c and Kantorovič [5] can be obtained in this setting by using Theorem 3.1 of this paper as Theorem 2.2 is used in [3].

7. Proof of Theorem 6.1. We show that Theorem 6.1 follows as a corollary of Theorem 3.1. To do so it is necessary to identify the subspace $S^{\prime}(\Sigma, X)$ and to construct the $\eta$ map of Theorem 3.1. The required space $S^{\prime}(\Sigma, X)$ is the space of simple functions which are representable as simple functions over $\left\{E_{i}\right\}_{i=1}^{n}$ where $\bigcup_{i=1}^{i} E_{i}$ is a closed set and where $\bigcup_{i=1}^{n} E_{i}=H$. Suppose $E \subset \Sigma$. Consider the directed set $\Lambda(E)=\{(F, V): F \subset E, F$ closed and $E \subset V, V$ open $\}$ where the direction is given by $(F, V)>\left(F^{\prime}, V^{\prime}\right)$ if and only if $F^{\prime} \subset F$ and $V \subset V^{\prime}$. Define the net of 
functions $\left\{\phi_{\alpha}\right\}_{\Lambda(E)}$ (using Urysohn's lemma) such that for $\alpha=(F, V), \phi_{\alpha}$ is a continuous function whose values are taken in $[0,1]$ which is 1 on $F$ and 0 on the complement of $V$. In the following lemma we establish the preliminaries necessary to define the $\eta$ map.

7.1 Lemma. Suppose $E$ and $\left\{\phi_{\alpha}\right\}_{\Lambda(E)}$ are as above. Then, for each vector $x \in X$, $\left\{\phi_{\alpha} \cdot x\right\}_{\Lambda(E)}$ is weakly Cauchy in CTB. Furthermore, if $\left\{\phi_{\alpha}\right\}_{\Lambda(E)}$ and $\left\{\psi_{\alpha}\right\}_{\Lambda(E)}$ are any two nets picked in the above fashion, then $\left\{\phi_{\alpha} \cdot x\right\}$ and $\left\{\psi_{\alpha} \cdot x\right\}$ are weakly Cauchy equivalent in $C T B$.

Proof. It is sufficient to show that $\left\{\phi_{\alpha}\right\}$ is weakly Cauchy in the space $C R$ of bounded continuous real valued functions on $H$. Suppose $c^{\prime} \in C R^{\prime}$. Then theorem [1, p. 262] implies there is a regular bounded additive set function $\mu_{c^{\prime}}$ such that $\left\langle c^{\prime}, f\right\rangle=\int f d \mu_{c^{\prime}}$. Therefore, given $\varepsilon>0$, there is a closed set $F_{\varepsilon} \subset E$ and an open set $V_{\varepsilon} \supset E$ such that $\|\mu\|\left(V_{\varepsilon} \mid E\right)$ and $\|\mu\|\left(E \backslash F_{\varepsilon}\right)$ are both smaller than $\varepsilon / 2$. If $(F, V)$, $\left(F^{\prime}, V^{\prime}\right)>\left(F_{\varepsilon}, V_{\varepsilon}\right)$, then

$$
\begin{aligned}
\|\mu\|\left(V \cup V^{\prime} \mid F \cap F^{\prime}\right) & \leqq\|\mu\|\left(V_{\varepsilon} \mid F_{\varepsilon}\right) \\
& =\|\mu\|\left(V_{\varepsilon} \mid E\right)+\|\mu\|\left(E \backslash F_{\varepsilon}\right)<\varepsilon / 2+\varepsilon / 2=\varepsilon .
\end{aligned}
$$

Therefore, for $\alpha=(F, V)$ and $\alpha^{\prime}=\left(F^{\prime}, V^{\prime}\right)$ and $\alpha, \alpha^{\prime}>\alpha_{\varepsilon}=\left(F_{\varepsilon}, V_{\varepsilon}\right), \phi_{\alpha}$ differs from $\phi_{\alpha^{\prime}}$ on a set of $\|\mu\|$-measure less than $\varepsilon$. Therefore, for $\alpha, \alpha^{\prime}>\alpha_{\varepsilon}$,

$$
\left|\left\langle c^{\prime}, \phi_{\alpha}\right\rangle-\left\langle c^{\prime}, \phi_{\alpha^{\prime}}\right\rangle\right|=\left|\int\left(\phi_{\alpha}-\phi_{\alpha^{\prime}}\right) d \mu\right|<\varepsilon,
$$

and it follows that $\left\{\left\langle c^{\prime}, \phi_{\alpha}\right\rangle\right\}$ is Cauchy. Hence, $\left\{\phi_{\alpha}\right\}$ is weakly Cauchy in $C R^{\prime}$. That $\left\{\phi_{\alpha}\right\}_{\Lambda(E)}$ and $\left\{\psi_{\alpha}\right\}_{\Lambda(E)}$ are weakly Cauchy equivalent follows by an analogous argument.

For each $E \in \Sigma$ and corresponding set of functions $\left\{\phi_{\alpha}\right\}_{\Lambda(E)}$, and each $x \in X$, we denote by $\chi(E, x)$ the weak limit of $\left\{\phi_{\alpha} \cdot x\right\}_{\Lambda(E)}$. Define the linear map $\eta$ from $S(\Sigma, X)+C T B$ into $(C T B)^{\omega}$ by $\eta(f)=f$ for $f \in C T B$ and $\eta\left(\sum_{i} \chi_{E_{i}} \cdot x_{i}\right)=\sum_{i} \chi\left(E_{i} \cdot x_{i}\right)$. Since the decomposition of a function into a continuous function and a simple function is unique modulo constant functions, the linear map $\eta$ is well defined. In the following lemma we establish that the $\eta$ map has the desired properties and thus complete the proof of 6.1 except for the uniqueness of $K$.

7.2 LEMMA. Suppose $\eta$ is as above.

(i) For each continuous seminorm $p$ on $X,|\eta|_{\rho_{p}}=1$.

(ii) The space CTB is a subset of the closure of $S^{\prime}(\Sigma, X)$ under the topology of uniform convergence.

(iii) The linear map $\eta$ is a continuous linear map from $S^{\prime}(\Sigma, X)+C T B$ into $(C T B)^{\omega}$.

Proof (i). We show that for each continuous seminorm $p$ on $X, \rho_{p}^{\prime \prime}\left(\eta\left(\sum_{i} \chi_{E_{i}} \cdot x_{i}\right)\right)$ $\leqq \rho_{p}\left(\sum_{i} \chi_{E_{i}} \cdot x_{i}\right)$. The simple function $\sum_{i=1}^{n} \chi_{E_{i}} \cdot x_{i}$ in $S(\Sigma, X)$ implies that there are nets $\left\{\phi_{i \alpha_{i}} \cdot x_{i}\right\}_{\Lambda\left(E_{i}\right)}, i=1, \ldots, n$, defined as in this section, which are weakly convergent to $\eta\left(\chi_{E_{i}} \cdot x_{i}\right), i=1, \ldots, n$, respectively. Let $\Lambda$ denote the directed set defined 
by $\Lambda\left(E_{1}\right) \times \Lambda\left(E_{2}\right) \times \cdots \times \Lambda\left(E_{n}\right)$. Define the nets $\left\{\phi_{i \alpha}\right\}, i=1, \ldots, n$, over $\Lambda$ by $\phi_{i \alpha}=\phi_{i\left(\alpha_{1}, \ldots, \alpha_{n}\right)}=\phi_{i \alpha_{i}}$. Then, $\left\{\phi_{i \alpha} \cdot x_{i}\right\}_{\Lambda}$ converges weakly to $\eta\left(\chi_{E_{i}} \cdot x_{i}\right)$. We now construct nets $\left\{h_{i \alpha}\right\}_{\Lambda}, i=1, \ldots, n$, in the fashion of Wayment [12] such that for each $\alpha, 0 \leqq \sum h_{i \alpha}(t) \leqq 1$ for $t \in H$ and such that, for each $i,\left\{h_{i \alpha} \cdot x_{i}\right\}_{\Lambda}$ converges weakly to $\eta\left(\chi_{E_{i}} \cdot x_{i}\right)$. For each $\alpha \in \Lambda$ define $h_{1 \alpha}=\phi_{1 \alpha}, h_{2 \alpha}=\phi_{2 \alpha}-\min \left(\phi_{2 \alpha}, h_{1 \alpha}\right)$, and in general $h_{j \alpha}=\phi_{j \alpha}-\min \left(\phi_{j \alpha}, \sum_{i=1}^{j-1} h_{i \alpha}\right)$. Then, $0 \leqq \sum_{i=1}^{n} h_{i \alpha}(t) \leqq 1$ for $t \in H$. Choose $j$. Then for fixed $\alpha, h_{j \alpha}$ differs from $\phi_{j \alpha}$ only on $\bigcup_{i \neq j}\left(V_{i} \cap V_{j}\right)$ where $\alpha=\left(\alpha_{1}, \ldots, \alpha_{n}\right)$ and $\alpha_{i}=\left(F_{i}, V_{i}\right), i=1, \ldots, n$. Suppose $c^{\prime} \in(C B R)^{\prime}$. As in 7.1 we let $\mu_{c^{\prime}}$ denote the measure corresponding to $c^{\prime}$. Then,

$$
\begin{aligned}
\left\|\mu_{c^{\prime}}\right\|\left(\bigcup_{i \neq j}\left(V_{i} \cap V_{j}\right)\right) & \leqq \sum_{i \neq j}\left\|\mu_{c^{\prime}}\right\|\left(V_{i} \cap V_{j}\right) \\
& =\sum_{i \neq j}\left\|\mu_{c^{\prime}}\right\|\left(\left(V_{i} \cap V_{j} \cap E_{i}\right) \cup\left(V_{i} \cap V_{j} \cap \widetilde{E}_{i}\right)\right) \\
& \leqq \sum_{i \neq j}\left[\left\|\mu_{c^{\prime}}\right\|\left(V_{j} \cap \widetilde{E}_{j}\right)+\left\|\mu_{c^{\prime}}\right\|\left(V_{i} \cap \widetilde{E}_{i}\right)\right] .
\end{aligned}
$$

The last inequality follows from the fact that $\left\|\mu_{c^{\prime}}\right\|$ is subadditive and the fact that $E_{i} \subset \widetilde{E}_{j}$, the complement of $E_{j}$. As $\alpha=\left(\alpha_{1}, \ldots, \alpha_{n}\right)$ runs through the directed set $\Lambda, \alpha_{i}=\left(F_{i}, V_{i}\right)$ runs through the directed set $\Lambda\left(E_{i}\right)$ for each $i$. Hence, for each $i$, $\left\|\mu_{c^{\prime}}\right\|\left(V_{i} \cap \tilde{E}_{i}\right)$ converges to zero. Therefore,

$$
\begin{aligned}
\lim _{\alpha}\left|\left\langle c^{\prime}, \phi_{h \alpha}-h_{j \alpha}\right\rangle\right| & =\lim _{\alpha}\left|\int\left(\phi_{j \alpha}-h_{j \alpha}\right) d \mu_{c^{\prime}}\right| \\
& \leqq \lim _{\alpha} \sup \left(\sum_{i \neq j}\left[\left\|\mu_{c^{\prime}}\right\|\left(V_{j} \cap \widetilde{E}_{j}\right)+\left\|\mu_{c^{\prime}}\right\|\left(V_{i} \cap \widetilde{E}_{i}\right)\right]\right)=0 .
\end{aligned}
$$

Hence, $\left\{\phi_{j \alpha}\right\}$ and $\left\{h_{j \alpha}\right\}$ are weakly Cauchy equivalent from which it follows that $\left\{h_{j \alpha} \cdot x_{j}\right\}_{\Lambda}$ converges weakly to $\phi\left(\chi_{E_{i}} \cdot x_{i}\right)$. Observe that

$$
\rho_{p}^{\prime \prime}\left(\eta\left(\sum_{i=1}^{n} \chi_{E_{i}} \cdot x_{i}\right)\right)=\sup \left\{\lim _{\alpha}\left|\left\langle c^{\prime}, \sum_{i=1}^{n} h_{i \alpha} \cdot x_{i}\right\rangle\right|: c^{\prime} \in B_{p}^{0}\right\}
$$

where $B_{p}^{0}=\left\{c^{\prime} \in(C T B)^{\prime}:\left|\left\langle c^{\prime}, f\right\rangle\right| \leqq 1\right.$ for all $f \in C T B$ such that $\left.\rho_{p}(f) \leqq 1\right\}$. Therefore,

$$
\begin{aligned}
\rho_{p}^{\prime \prime}\left(\eta\left(\sum_{i=1}^{n} \chi_{E_{i}} \cdot x_{i}\right)\right) & \leqq \limsup _{\alpha} \rho_{p}\left(\sum_{i=1}^{n} h_{\alpha i} \cdot x_{i}\right) \\
& =\limsup _{\alpha}\left(\sup _{t \in H} p\left(\sum_{i=1}^{n} h_{\alpha i}(t) \cdot x_{i}\right)\right) \\
& \leqq \limsup _{\alpha}\left(\left(\sup _{t \in H} \sum_{i=1}^{n} h_{\alpha i}(t)\right)\left(\max _{1 \leqq i \leqq n} p\left(x_{i}\right)\right)\right) \\
& =\max _{1 \leqq i \leqq n} p\left(x_{i}\right)=\rho_{p}\left(\sum_{i=1}^{n} \chi_{E_{i}} \cdot x_{i}\right) .
\end{aligned}
$$


Proof of (ii). Suppose $f \in C T B$ and suppose $U$ is an open neighborhood of $\theta_{X}$. Since the range of $f$ is totally bounded, there exists a finite number of points $\left\{x_{i}\right\}_{i=1}^{n}$ such that $\left\{\frac{1}{2} U+x_{i}\right\}_{i=1}^{n}$ covers the range of $f$. Hence $\left\{\frac{1}{2} \bar{U}+x_{i}\right\}_{i=1}^{n}$ covers the range of $f$. Let $F_{i}=f^{-1}\left[\frac{1}{2} \bar{U}+x_{i}\right]$ for each $i$. Define $E_{1}=F_{1}, E_{2}=F_{2}-E_{1}, E_{3}=F_{3}$ $-E_{1} \cup E_{2}$, and in general let $E_{j}=F_{j}-\bigcup_{i=1}^{j-1} E_{i}$. Finally, let $S_{U}=\sum f\left(t_{i}\right) \chi_{E_{i}}$ where $t_{i} \in E_{i}$. We note that $S_{U} \in S^{\prime}(\Sigma, X)$ and $S_{U}(t)-f(t) \in U$ for each $t$.

Proof of (iii). Our plan is to use Lemma 3.2 to establish this result. In order to do so, it is convenient to establish the following sublemma, which extends the corresponding results in [3] and [11].

7.3 Sublemma. Suppose $S$ is a locally convex space. A sufficient condition to guarantee that a net $\left\{s_{\alpha}^{\omega}\right\}_{\Lambda}$ in $S^{\omega}$ converges to $s \in S$ is that for each $\alpha$ there is a net $\left\{s_{\alpha, \beta}\right\}_{\beta \in \Lambda(\alpha)}$ such that

(a) for each $\alpha \in \Lambda,\left\{s_{\alpha, \beta}\right\}_{\beta \in \Lambda(\alpha)}$ converges weakly to $s_{\alpha}^{\omega}$,

(b) $s_{\alpha, \beta}-s$ converges to $\theta_{S}$ as $\alpha$ runs through the directed set $\Lambda$.

Proof. It is sufficient to show that for each continuous seminorm $\rho$ on $S$ that $\rho^{\prime \prime}\left(s_{\alpha}^{\omega}-s\right) \rightarrow 0$. Choose $\varepsilon>0$. Since $s_{\alpha, \beta}-s$ converges to $\theta_{S}$ then there is an $\alpha_{0} \in \Lambda$ such that $\alpha>\alpha_{0}$ implies $\rho\left(s_{\alpha, \beta}-s\right)<\varepsilon$. Choose $\alpha>\alpha_{0}$. Then

$$
\rho^{\prime \prime}\left(s_{\alpha}^{\omega}-s\right)=\sup \left\{\lim _{\beta}\left|\left\langle s^{\prime}, s_{\alpha, \beta}-s\right\rangle\right|: s^{\prime} \in B_{\rho}^{0}\right\} \leqq \lim _{\beta} \sup \rho\left(s_{\alpha, \beta}-s\right)<\varepsilon .
$$

We continue with the proof of (iii). Suppose $\left\{s_{\alpha}\right\}_{\Lambda}=\left\{\sum_{i=1}^{n(\alpha)} \chi_{E_{i}^{\alpha}} \cdot x_{i}^{\alpha}\right\}$ is a net in $S^{\prime}(\Sigma, X)$ which converges to $f \in C T B$ in the topology of uniform convergence. Our plan is to construct for each $\alpha \in \Lambda$ a net $\left\{s_{\alpha, \beta}\right\}_{\beta \in \Lambda(\alpha)}$ which converges weakly to $\eta\left(s_{\alpha}\right)$ so that Sublemma 7.3 is satisfied in order to apply Lemma 3.2. Fix $\alpha$. Since $s_{\alpha} \in S^{\prime}(\Sigma, X), A_{j}^{\alpha}=\bigcup_{i=1}^{j} E_{i}^{\alpha}$ is a closed set. For each $j$ we define the directed set

$$
\Lambda\left(A_{j}^{\alpha}\right)=\left\{\left(A_{j}^{\alpha}, V\right): V \text { is an open set containing } A_{j}^{\alpha}\right\}
$$

where the direction is given by inclusion. For each $j$ we define a net of continuous functions $\left\{\phi_{j, \beta_{j}}\right\}_{\beta_{j} \in \Lambda\left(A_{j}^{\alpha}\right)}$ such that, for each $j$ and for $\beta_{j}=\left(A_{j}^{\alpha}, V\right), \phi_{j, \beta_{j}}$ takes its values in $[0,1]$, is 1 on $A_{j}^{\alpha}$ and is 0 on $\tilde{V}$. Then for each $j,\left\{\phi_{j, \beta_{j}}\right\}_{\beta_{j} \in \Lambda\left(A_{j}^{\alpha}\right)}$ converges in measure to $\chi_{A_{j}^{\alpha}}$ for every bounded regular finitely additive set function on $\Sigma$, and hence is weakly Cauchy in $C B R$. Define the directed set $\Lambda(\alpha)$ to be $\Lambda\left(A_{1}^{\alpha}\right) \times \cdots \times \Lambda\left(A_{n(\alpha)}^{\alpha}\right)$. For each $j$ we define the net of open sets $\left\{V_{j \beta}\right\}_{\beta \in \Lambda(\alpha)}$ by $V_{j \beta}=V$ where $\beta_{j}=\left(A_{j}^{\alpha}, V\right)$ for $\beta=\left(\beta_{1}, \ldots, \beta_{j}, \ldots, \beta_{n(\alpha)}\right)$. For each $\beta \in \Lambda(\alpha)$ define $\left\{h_{j \beta}\right\}_{j=1}^{n(\alpha)}$ by $h_{1 \beta}=\phi_{1 \beta}$ and $h_{j \beta}=\phi_{j \beta}-\min \left(\phi_{j \beta}, \sum_{i=1}^{j=1} h_{i \beta}\right)$. Then for each $\beta \in \Lambda(\alpha),\left\{h_{j \beta}\right\}_{j=1}^{n(\alpha)}$ is a partition of unity subordinate to $\left\{V_{j \beta}\right\}_{j=1}^{n(\alpha)}$. Next we show that, for each $j,\left\{h_{j \beta}\right\}_{\beta \in \Lambda(\alpha)}$ converges in measure to $\chi_{E_{j}}$ for every bounded regular finitely additive set function on $\Sigma$. Suppose $\mu$ is such a set function and suppose $\varepsilon>0$. For each $\beta, h_{j \beta}$ differs from $\chi_{E_{j}}$ on $\left(E_{j}^{\alpha} \cap\left(\bigcup_{i=1}^{j=1} V_{i \beta}\right)\right) \cup\left(V_{j \beta} \mid A_{j}^{\alpha}\right)$. For each $i<j,\|\mu\|\left(E_{j}^{\alpha} \cap V_{i \beta}\right)<\|\mu\|\left(\tilde{A}_{i}^{\alpha} \cap V_{i \beta}\right)$ which converges to zero as $\beta$ runs through $\Lambda(\alpha)$ because of the regularity of $\mu$ and because of the manner in which $\left\{V_{j \beta}\right\}_{\beta \in \Lambda(\alpha)}$ is constructed. For the same reason, 
$\|\mu\|\left(V_{j \beta} \backslash A_{j}^{\alpha}\right)$ converges to zero. Hence, it follows that $\left\{h_{j \beta}\right\}_{\beta \in \Lambda(\alpha)}$ converges to $\chi_{E_{j}^{\alpha}}$ in measure for all bounded regular finitely additive set functions. Hence, it follows that for each $i,\left\{h_{i \beta} \cdot x_{i}^{\alpha}\right\}_{\beta \in \Lambda(\alpha)}$ converges weakly to $\chi\left(E_{i}^{\alpha}, x_{i}^{\alpha}\right)$ and hence that $\left\{\sum_{i=1}^{n(\alpha)} h_{i \beta} \cdot x_{i}^{\alpha}\right\}_{\beta \in \Lambda(\alpha)}$ converges weakly to $\sum_{i=1}^{n(\alpha)} \chi\left(E_{i}^{\alpha}, x_{i}^{\alpha}\right)=\eta\left(\sum_{i=1}^{n(\alpha)} \chi_{E_{i}^{\alpha}} \cdot x_{i}^{\alpha}\right)$. For each $\beta \in \Lambda(\alpha)$ define $s_{\alpha, \beta}=\sum_{i=1}^{n(\alpha)} h_{i \beta} \cdot x_{i}^{\alpha}$. Then, $\left\{s_{\alpha, \beta}\right\}_{\beta \in \Lambda(\alpha)}, \alpha \in \Lambda$, satisfies condition (a) of Sublemma 7.3. Suppose $p$ is a continuous seminorm on $X$ and suppose $\varepsilon>0$. Since $\left\{s_{\alpha}\right\}_{\Lambda}$ converges to $f$ in the topology of uniform convergence, it follows that there is an $\alpha_{0}$ such that $\alpha>\alpha_{0}$ implies $\rho_{p}\left(s_{\alpha}-f\right)<\varepsilon$. Suppose $\alpha>\alpha_{0}$. Then

$$
\begin{aligned}
\rho_{p}\left(s_{\alpha, \beta}-f\right) & =\sup _{t \in H} p\left(f(t)-\sum h_{i \beta}(t) \cdot x_{i}^{\alpha}\right) \\
& =\max _{1 \leqq i \leqq n(\alpha)} \sup _{t \in E_{i}^{\alpha}} p\left(\sum h_{i \beta}(t)\left(f(t)-x_{i}^{\alpha}\right)\right) \\
& =\max _{1 \leqq i \leqq n(\alpha)} \sup _{t \in E_{i}^{\alpha}} p\left(f(t)-x_{i}^{\alpha}\right)=\rho_{p}\left(s_{\alpha}-f\right)<\varepsilon .
\end{aligned}
$$

Hence it follows that $\lim _{\alpha} \rho_{p}\left(s_{\alpha, \beta}-f\right)=0$. Therefore, $\lim _{\alpha} s_{\alpha, \beta}-s=\theta_{S}$, from which it follows that (b) of Sublemma 7.3 is satisfied. Hence, $\left\{\eta\left(s_{\alpha}\right)\right\}_{\Lambda}$ converges to $f$ in $C T B$. Therefore, Lemma 3.2 implies $\eta$ is continuous on $S^{\prime}(\Sigma, X)+C T B$.

7.4 LEMMA. Suppose $K_{1}$ and $K_{2}$ are weakly regular and give the same transformation on $C T B$. Then $K_{1}(E)=K_{2}(E)$ for all $E \in \Sigma$.

Proof. For each $y^{\prime} \in Y^{\prime}$ and $x \in X,\left\langle y^{\prime},[K(\cdot)](x)\right\rangle$ is a bounded regular finitely additive set function on $\Sigma$ and generates a continuous linear functional on $C B R$. Theorem [1, p. 262] implies such set functions are unique. Thus, if $K_{1}$ and $K_{2}$ generate the same transformation on $C T B$, then for each $y^{\prime}, x,\left\langle y^{\prime},\left[K_{1}(\cdot)-K_{2}(\cdot)\right](x)\right\rangle$ generates the zero functional on $C B R$. Hence for each $E,\left[K_{1}(E)-K_{2}(E)\right](x)=\theta_{Y}$ which in turn implies $K_{1}(E)-K_{2}(E)=\theta_{L\left[X, Y^{\omega}\right]}$.

8. Proofs of Theorems 6.2 and 6.3. The proofs in this section mirror those of $\$ 7$ and hence only brief indications of proofs are given in this section. As before, our plan is to use Theorem 3.1, and therefore, we must identify the map $\eta$ and the collection $S^{\prime}\left(\Sigma_{c}, X\right)$. In this setting $S^{\prime}\left(\Sigma_{c}, X\right)$ denotes the space of simple functions $\sum_{i=1}^{n} \chi_{E_{i}} \cdot x_{i}$ such that $\bigcup_{i=1}^{j} E_{i}$ is a compact subset of $H$ for $j<n$. Let $K$ be a compact subset of $H$ and let $\Sigma_{K}$ denote the field generated by the closed subsets of $K$. Then $\bigcup_{K \subset H} \Sigma_{K}$ is a ring in $H$ which contains all of the compact sets in $H$. Hence if $\Sigma_{c}$ denotes the ring generated by the compact subsets of $H$, we have $\Sigma_{c} \subset \bigcup_{K \subset H} \Sigma_{K}$. On the other hand if $F \in \bigcup_{K \subset H} \Sigma_{K}$, then $F \in \Sigma_{K}$ for some $K$. We observe that $\mathfrak{A}_{K}=\left\{E \cap K \mid E \in \Sigma_{c}\right\}$ is a field of sets satisfying $\mathfrak{A}_{K} \subset \Sigma_{c}$ which contains all the closed sets in $K$. Hence $\Sigma_{K} \subset \mathfrak{A}_{K}$. This implies $F \in \mathfrak{A}_{K}$ and consequently $\Sigma_{c}$ $=\bigcup_{K \subset H} \Sigma_{K}$. It follows that for each $E$ in $\Sigma_{c}$ there is an open set $O_{E}$ containing $E$ which has compact closure. Define the directed set $\Lambda(E)$ by

$$
\Lambda(E)=\left\{(F, V): F \subset E \text { is closed, } E \subset V \subset O_{E} \text { is open }\right\}
$$


where the direction is given by $\left(F^{\prime}, V^{\prime}\right)>(F, V)$ means $F \subset F^{\prime}$ and $V^{\prime} \subset V$. For each $\alpha=(F, V) \in \Lambda(E)$ define (using Urysohn's lemma) a continuous function $\phi_{\alpha}$ which has its range in $[0,1]$ and such that $\phi_{\alpha} \equiv 1$ on $F$ and $\phi_{\alpha} \equiv 0$ on $\tilde{V}$. Suppose $T$ is a linear functional on $C_{c} R$. As in [8, p. 139], it is possible to decompose $T$ into the difference of two positive linear functions and hence by Theorem 2.14 [9, p. 40] there is a unique Borel measure which is outer regular and inner regular on Borel sets of compact closure such that $T(f)=\int f d \mu$ for $f \in C_{c} R$. Then proceeding as in the proof of Lemma 7.1, we have the following lemma.

8.1 Lemma. For each $E \in \Sigma_{c}$, the net $\left\{\phi_{\alpha}\right\}_{\alpha \in \Lambda(E)}$ is weakly convergent. Furthermore, if $\Lambda^{\prime}(E)$ is a directed set which is cofinal with $\Lambda(E)$ and the net $\left\{\psi_{\alpha}\right\}_{\alpha \in \Lambda^{\prime}(E)}$ is constructed in a fashion analogous to that of $\left\{\phi_{\alpha}\right\}_{\alpha \in \Lambda(E)}$ then the two nets are weakly Cauchy equivalent.

For each $E$, since $\left\{\phi_{\alpha}\right\}_{\alpha \in \Lambda(E)}$ is weakly Cauchy in $C_{c} R$, then $\left\{\phi_{\alpha} \cdot x\right\}_{\alpha \in \Lambda(E)}$ is weakly Cauchy in $C_{c}$ for each $x \in X$. As before, we let $\chi(E, x)$ denote the weak limit of $\left\{\phi_{\alpha} \cdot x\right\}_{\alpha \in \Lambda(E)}$. Define the linear map $\eta$ from $C_{c}+S\left(\Sigma_{c}, X\right)$ into $C_{c}^{\omega}$ by $\eta\left(f+\sum_{\mathfrak{i}} \chi_{E_{\mathfrak{i}}} \cdot x_{\mathfrak{i}}\right)$ $=f+\sum_{i} \chi\left(E_{i}, x_{i}\right)$ for $f \in C_{c}$ and $\sum_{i} \chi_{E_{i}} \cdot x_{i} \in S\left(\Sigma_{c}, X\right)$.

8.2 Lemma. Suppose $\eta$ and $S^{\prime}\left(\Sigma_{c}, X\right)$ are as above. Then,

(i) the space $C_{c}$ is in the closure of $S^{\prime}\left(\Sigma_{c}, X\right)$ under the topology of uniform convergence,

(ii) the linear map $\eta$ is continuous on the space $C_{c}+S^{\prime}\left(\Sigma_{c}, X\right)$,

(iii) the linear map $\eta$ is continuous on the space $S\left(\Sigma_{c}, X\right)$.

Furthermore, for each continuous seminorm $p$ on $X,|\eta|_{\rho_{p}}=1$.

The proof of this lemma follows as the proof of Lemma 7.2 by using the above characterization of linear functions and by observing that in this setting the function $\phi_{\alpha}$ can be constructed to have compact support.

Theorem 6.2 now follows from Theorem 3.1 except for the uniqueness of $K$. However, the uniqueness of $K$ follows from Theorem 2.14 [9, p. 40] in the same way the uniqueness of $K$ was obtained using Theorem 2 [1, p. 262].

Theorem 6.3 now follows from Theorems 6.2 and 4.7 since $C_{0}$ is the closure of $C_{c}$ in the topology of uniform convergence.

9. Proofs of Theorems 6.5 and 6.6. Theorem 6.5 is first proved for $C_{c}$ under the topology of uniform convergence on compact subsets. We remark that this topology is a weaker topology than that used in 6.2. Also note that the set function $K$ in Theorems 6.5 and 6.6 is of bounded semivariation on a ring and cannot in general be extended to a set function of bounded semivariation on the field generated by the ring.

9.1 Lemma. Suppose $H$ and $\Sigma_{c}$ are as in Theorem 6.2. If $T$ is a continuous linear map from $C_{c}$ into $Y$, then there is a unique finitely additive set function $K$ on $\Sigma_{c}$ with values in $L\left[X, Y^{\omega}\right]$ which is of bounded $\left(p, q^{\prime \prime}\right)$ variation with compact $q^{\prime \prime}$-support such that $T(f)=\int d K \cdot f$. Furthermore, if $T$ is $(\rho, q)$ related then $W K_{\rho, q^{\prime \prime}}=|T|_{\rho, q}$. 
Proof. Define $S^{\prime}\left(\Sigma_{c}, X\right)$ as in $\S 8$ and for each $E \in \Sigma_{c}$ construct the net $\left\{\phi_{\alpha}\right\}_{\alpha \in \Lambda(E)}$ as in that section. That $\left\{\phi_{\alpha}\right\}_{\alpha \in \Lambda(E)}$ is weakly Cauchy follows from Theorem 4.10.1 in $[4$, p. 203]. Define $\eta$ as in $\S 8$. That Lemma 8.2 is true in this setting follows as indicated in $\S 8$ using Theorem 4.10.1 in [4] in place of Theorem 2.14 in [9]. Hence, it follows from Lemma 3.2 that there is a set function $K$ on $\Sigma_{c}$ with values in $L\left[X, Y^{\omega}\right]$ which is quasi-Gowurin with respect to the topology of uniform convergence on compact subsets such that, for $f \in C_{c}, T(f)=\int d K \cdot f$ and such that if $T$ is $(\rho, q)$ related, then $W K_{\rho, q^{\prime \prime}}=|T|_{\rho, q}$. This implies given a continuous seminorm $q$ on $Y$, there is a compact set $M_{q}$ and a continuous seminorm $p$ on $X$ such that

$$
\begin{aligned}
q^{\prime \prime}\left(\sum\left[K\left(E_{i}\right)\right]\left(x_{i}\right)\right) & \leqq W K_{\rho, q^{\prime \prime}} \sup _{t \in M_{q}} p\left(\sum \chi_{E_{i}}(t) \cdot x_{i}\right) \\
& =W K_{\rho, q^{\prime \prime}} \max _{i}\left\{p\left(x_{i}\right): E_{i} \cap M_{q} \neq \varnothing\right\} .
\end{aligned}
$$

Therefore, for each $x$ in $X, q^{\prime \prime}([K(E)](x))$ has as support $M_{q}$. Hence, $K$ is of bounded $\left(p, q^{\prime \prime}\right)$ variation with compact $q^{\prime \prime}$-support. The uniqueness of $K$ follows as in $\S 7$.

Theorem 6.5 now follows from Theorem 4.7 since $C$, the space of all continuous functions, is the completion of $C_{c}$, the space of continuous functions of compact support, both with the topology of uniform convergence on compact subsets. If $Y$ is a normed space, then $K$ has compact support $M$, which implies all functions in $C$ are $K$ integrable and that $\int d K \cdot f=\int d K \cdot f_{M}$ where $f_{M}$ denotes the restriction of $f$ to $M$. Also $E \int d K \cdot f=E \int d K \cdot f_{M}$. Lemma 4.5 implies $E \int d K \cdot f_{M}=\int d K \cdot f_{M}$ from which Theorem 6.6 follows.

\section{Concluding remarks.}

10.1 Remark. In order to hypothesize the existence of the $\eta$ map in Theorem 3.1 , it is necessary that the dual $F^{\prime}$ of the function space $F$ separate points of $F$ (which is assured when $F$ has a locally convex topology). This is sufficient to guarantee that $F$ can be imbedded in $F^{\prime \prime}$. Under this additional hypothesis we may retain the view that $F \subset F^{\omega}$ under the topology of uniform convergence on equicontinuous subsets of $F^{\prime}$ when $X$ and $Y$ are topological vector spaces. Hence, Theorem 3.1 is valid in this more general setting.

10.2 Remark. A sufficient condition to obtain the representation theorem of Theorem 3.1 is that $T$ has a continuous extension to $F+S(\Sigma, X)$. A seemingly weaker condition is that the $\eta$ map of Theorem 3.1 exists. It is interesting to note that the existence of the $\eta$ map implies the representation theorem which in turn implies that the composite map $T^{\omega} \eta$ (an extension of $T$ ) is continuous. The classical integral representations for functionals on real function spaces follow from Theorem 3.1 using this observation and the Hahn-Banach theorem.

10.3 Remark. The set functions in Theorems 6.2, 6.3, 6.5, and 6.6 all can be constructed to assume values in $L\left[X, Y^{+}\right]$rather than $L\left[X, Y^{\omega}\right]$ by using the techniques of the proof in [3] and by changing $\Sigma_{c}$ to be the ring of sets generated 
by the condition that, for each $E$ in $\Sigma, \chi_{E}$ is the pointwise limit of a sequence of uniformly bounded continuous functions of uniform compact support.

ADDENDUM. The authors have been interested in obtaining conditions sufficient to insure that $F^{\prime}$ separates points of $F$ (Remark 10.1). Motivated by the abstract (Abstract 672-422, Notices Amer. Math. Soc. 17 (1970), 203) of A. H. Shuchat which came to the authors' attention after the preceding manuscript was prepared, we obtain the following results.

Lemma. Suppose $F$ is a topological vector space of functions with topology $\tau$ from an arbitrary space $H$ into a topological vector space $X$ and $T$ is a continuous linear operator from $F$ into the topological vector space $Y$. Suppose further that $Y^{\prime}$ separates $T[f]$. Let $F_{T}=F / \operatorname{Ker}(T)$ under the inherited topology. Then $F_{T}^{\prime}$ separates points in $F_{T}$.

REMARK. If $Y^{\prime}$ separates points in $Y$, then $F_{T}^{\prime}$ separates points in $F_{T}$ for all $T$.

Hence, if $F \subset \Gamma$ and the topology $\tau$ on $F$ can be extended to the simple functions, then it is possible to hypothesize the existence of an $\eta$ map analogous to the one in Theorem 3.1 from $F_{T}+S_{T}(\Sigma, X)$ into $F_{T}^{\omega}$. Here again, $\Gamma$ is the closure under the uniform topology of the simple functions $S(\Sigma, X)$ over a ring $\Sigma$ of subsets of $H$ and $\tau$ is a topology not stronger than the topology of uniform convergence, and $S_{T}(\Sigma, X)=S(\Sigma, X) /(\operatorname{Ker}(T) \cap S(\Sigma, X))$.

Theorem. Suppose $H, \Sigma, X, Y, F, \tau, S(\Sigma, X), \Gamma$, and $T$ are as above. Suppose further that there is a subspace $S^{\prime}(\Sigma, X)$ in $S(\Sigma, X)$ such that

(i) $F$ is contained in the $\tau$ closure of $S^{\prime}(\Sigma, X)$, and

(ii) there is a linear map $\eta_{T}$ from $F_{T}+S_{T}(\Sigma, X)$ into $F_{T}^{\omega}$ such that $\eta_{T}$ restricted to each of $S_{T}$ and $F_{T}+S_{T}^{\prime}(\Sigma, X)$ is continuous.

Then there is a set function $K$ on $\Sigma$ with values in $L\left[X, Y^{\omega}\right]$ which is quasi-Gowurin with respect to $\tau$ and such that, for each $f \in F, T(f)=\int d K \cdot f$.

Theorems 6.1, 6.2, 6.3, 6.5, and 6.6 now follow for the case that $X$ and $Y$ are topological vector spaces and $Y^{\prime}$ separates points of $Y$.

\section{REFERENCES}

1. N. Dunford and J. Schwartz, Linear operators. I: General theory, Pure and Appl. Math., vol. 7, Interscience, New York, 1958. MR 22 \#8302.

2. R. J. Easton and D. H. Tucker, A generalized Lebesgue-type integral, Math. Ann. 181 (1969), 311-324.

3. J. R. Edwards and S. G. Wayment, $A$ unifying representation theorem, Math. Ann. 187 (1970), 317-328.

4. R. E. Edwards, Functional analysis. Theory and applications, Holt, Rinehart and Winston, New York, 1965. MR 36 \#4308.

5. I. G. Fihtengol'c and L. Kantorovič, Sur les opérations linéaires dans l'espace des fonctions bornées, Studia Math. 5 (1934), 69-98.

6. R. K. Goodrich, A Riesz representation theorem in the setting of locally convex spaces, Trans. Amer. Math. Soc. 131 (1968), 246-258. MR 36 \#5731. 
7. T. H. Hildebrandt, On bounded linear functional operations, Trans. Amer. Math. Soc. 36 (1934), 868-875.

8. M. A. Naĭmark, Normed rings, GITTL, Moscow, 1956; English transl., Noordhoff, Groningen, 1959. MR 19, 870; MR 22 \#1824.

9. W. Rudin, Real and complex analysis, McGraw-Hill, New York, 1966. MR 35 \#1420.

10. K. Swong, A representation theory of continuous linear maps, Math. Ann. 155 (1964), 270-291; errata, ibid. 157 (1964), 178. MR 29 \#2642.

11. D. J. Uherka, Generalized Stieltjes integrals and a strong representation theorem for continuous linear maps on a function space, Math. Ann. 182 (1969), 60-66. MR 40 \#705.

12. S. G. Wayment, Absolute continuity and the Radon theorem, Ph.D. Thesis, University of Utah, Salt Lake City, Utah, 1968.

Utah State University,

LOGAN, UTAH 84321 